
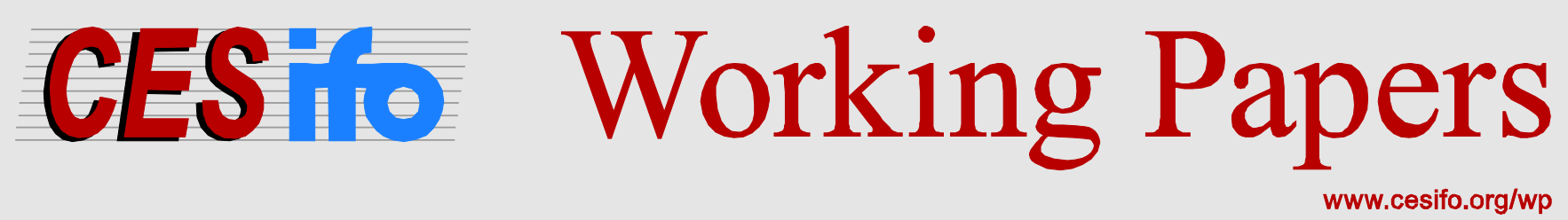

\title{
The Singular Value Decomposition of a Technology Matrix
}

\author{
Eric O’N. Fisher
}

\author{
CESIFO WORKING PAPER NO. 4566 \\ CATEGORY 12: EMPIRICAL AND THEORETICAL METHODS \\ JANUARY 2014
}

An electronic version of the paper may be downloaded

- from the SSRN website:

- from the RePEc website:

- from the CESifo website:

www.SSRN.com

Www.RePEc.org

www.CESifo-group.org/wp

\section{CESifo}




\title{
The Singular Value Decomposition of a Technology Matrix
}

\begin{abstract}
This paper is the first application of the singular value decomposition in general equilibrium theory. Every technology matrix can be decomposed into three parts: (1) a definition of composite commodities; (2) a definition of composite factors; and (3) a simple map of composite factor prices into composite goods prices. This technique gives an orthogonal decomposition of the price space into two complementary subspaces: (1) vectors that generate the price cone; and (2) a basis that describe the flats on the production possibility frontier. This decomposition can be used easily to compute Rybczynski effects.
\end{abstract}

JEL-Code: F100, D500.

Keywords: singular value decomposition, international trade, Rybezynski, StolperSamuelson.

\author{
Eric O’N. Fisher \\ Department of Economics \\ California Polytechnic State University \\ 1 Grand Avenue \\ USA - 93407 San Luis Obispo CA \\ efisher@calpoly.edu
}

December 12, 2013

I would like to thank an anonymous referee, Tanner Starbard and seminar participants at the Econometric Society Australasian Meetings at the University of Sydney and the Midwest International Economics Group at the University of Michigan for comments on an earlier draft. I owe a great debt of gratitude to Bill Ethier, whose scholarship has inspired me, whose conversations have shaped this manuscript, and whose friendship survived a star-crossed trip to the summit of Mt. Fuji. 


\section{Introduction}

A technology matrix is a description of the direct and indirect factor inputs that minimize unit costs, given local factor prices. If there are constant returns to scale, these requirements are independent of the quantities of output. Let $w$ be the vector of local factor costs. Then a technology is a mapping:

$$
w \rightarrow A(w) .
$$

It is customary to write this rule as an $n \times f$ matrix whose element $a_{i j}(w)$ is the cost-minimizing unit input requirement of factor $j$ in the production of $\operatorname{good} i$. In the textbook case with two goods and two factors, this rule has dimension four, but in most empirical applications, the number of goods is near $n \approx 60$ and the number of factors is near $f \approx 5$, so the mapping has dimension approximately $n f \approx 300$. In this paper, I will present a novel technique that summarizes the local information in a technology matrix succinctly.

This technique is used routinely in the transmission of images by computers. Here is a simple visual application that makes my point. Figure 1 is a file of 2800 kilobytes, and Figure 2 is one of size 90 kilobytes. Even though the compressed file is $98 \%$ smaller than the original, the image is quite readily recognizable as the same economist; the Philadelphia Museum of Art and the spring foliage around the Schuylkill are still clear in the picture with lower resolution. If it is costly to transmit information or if the original file consists of a true depiction and some noise, then it pays to separate the wheat from the chaff.

What does it mean to compress the information inherent in a technology matrix? Consider a matrix with $n=61$ goods and $f=5$ factors:

$$
A(w)=\left[\begin{array}{ccccc}
1 & 2 & 3 & 4 & 5 \\
10 & 20 & 30 & 40 & 50 \\
\vdots & \vdots & \vdots & \vdots & \vdots \\
590 & 1080 & 1770 & 2360 & 2950 \\
600 & 1200 & 1800 & 2400 & 3001
\end{array}\right]
$$

It has dimension 305, a size typical of technology matrices in empirical work. The small "error" in the lower right corner gives this matrix full rank two, but it is obvious that every sector is using factors in fixed proportions

$$
v^{T}=(1,2,3,4,5)
$$




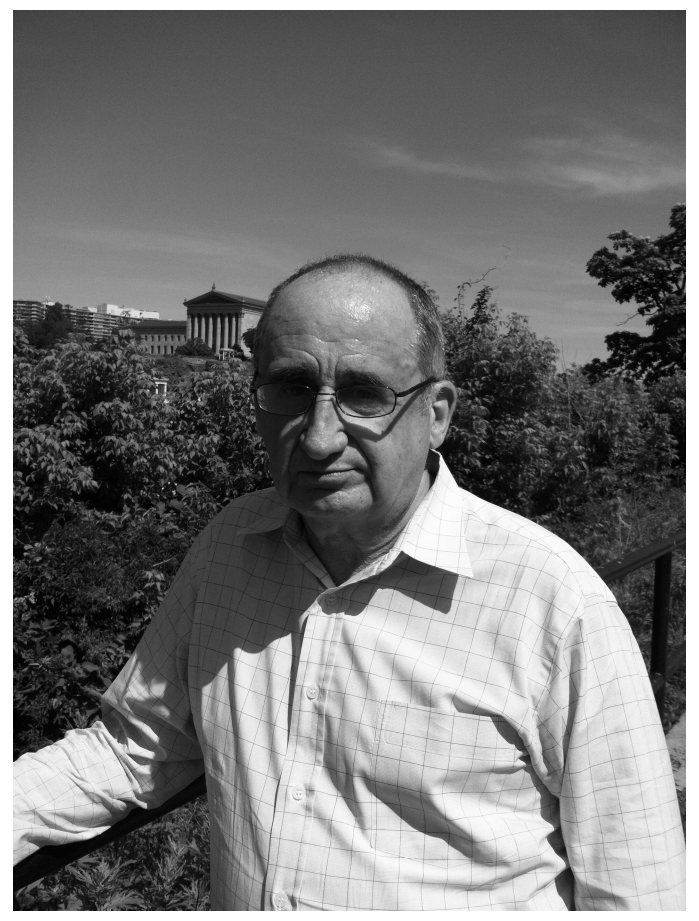

Figure 1: The full file with 2800kb

and that the sectors differ by the inverses of total factor productivities

$$
u=(1,10,20, \ldots, 600)^{T} .
$$

The singular value decomposition transmits all the information inherent in this matrix-including the minor "error"-by four vectors consisting of $2(n+f)=$ $2 *(5+61)=132$ elements. These vectors have a natural interpretation for a trade theorist; exploring the meaning of this decomposition is my contribution.

It should not be gainsaid that the matrix itself can be reduced by a factor of $56 \%$ without any loss of information. Almost all the power of the matrix is in the row vector $v^{T}$ that shows factor uses and the column vector $u$ related to total factor productivities. The representation

$$
A(w) \approx u v^{T}
$$

requires only $n+f=66$ elements. The approximation $u v^{T}$ has rank one, and it is computed with less than $22 \%$ of the original data. In international comparisons, 


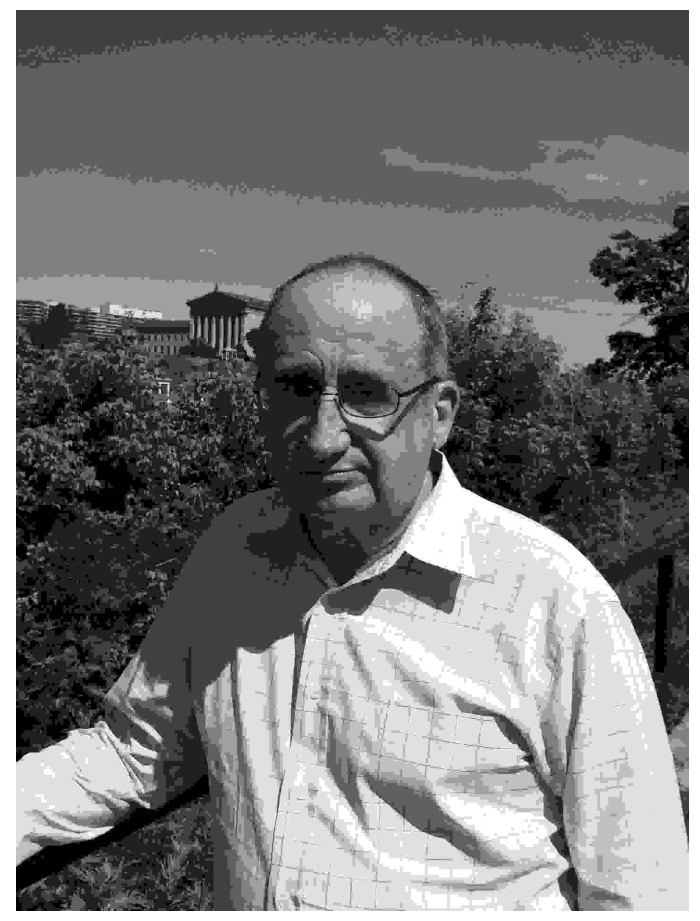

Figure 2: The compressed file with $90 \mathrm{~kb}$

the representation lends itself to an interpretation as factor augmenting technical differences and total factor productivity differences by sector.

To the best of my knowledge, this technique is completely novel in economics. Still, it is inspired by the span of Ethier's scholarship. Perhaps no other economist of his generation has thought as deeply about multi-dimensional issues in trade theory. He adumbrated the now standard pedagogical taxonomy of the four central results of Heckscher-Ohlin theory: (1) the Heckscher-Ohlin Theorem itself; (2) the Factor Price Equalization Theorem; (3) the Stolper-Samuelson Theorem, and (4) the Rybczynski Theorem. The profession now knows that these theorems concern the structure of technology matrices and their generalized inverses, but we have achieved these insights because of the seminal contributions Ethier (1974) and Ethier (1984). In fact, the simple example above has an elegant interpretation as an aggregate production function that uses five factors and jointly produces sixty-one outputs; although this insight draws on Chang et al. (1980), it will not be pursued below and may be a fruitful avenue for future research. In sum, I could have done none of this work if I had not stood on Ethier's shoulders. 


\section{The Singular Value Decomposition}

Let $A(w)$ be an $n \times f$ technology matrix, where $n$ is the number of goods, $f$ is the number of factors, and $w$ is an $f \times 1$ vector of factor prices. The canonical element of such a matrix $a_{i j}(w) \geq 0$ is the unit input requirement of factor $j$ in the production of good $i$. I assume that every sector exhibits constant returns to scale, and these requirements are independent of the quantities.

Taking factor prices as fixed, we may write $A(w)=A$. Implicit in much of the discussion is the idea that every technology behaves locally like a Leontief technology. Hence the links between factor prices and goods prices are true for all local price changes because we restrict ourselves to linear relationships. Likewise, the quantity effects are true for fixed factor prices, an assumption inherent in the definition of a Rybczynski effect.

The singular value decomposition of $A$ is: ${ }^{1}$

$$
A=U S V^{T}
$$

where the columns of the $n \times n$ matrix $U$ are the normalized eigenvectors of $A A^{T}$, the columns of $f \times f$ matrix $V$ are those of $A^{T} A$, and $n \times f$ matrix $S$ is a diagonal matrix, whose non-zero elements are the square roots of the eigenvalues of either $A A^{T}$ or $A^{T} A$. These eigenvalues are non-negative because both $A^{T} A$ and $A A^{T}$ are symmetric and positive semidefinite. It is customary to rank the diagonal elements of $S$ in decreasing in order and to permute the columns of $U$ and $V$ accordingly.

There are three cases: (1) there are more goods than factors $(n>f)$; (2) the even case $(n=f)$; and (3) there are fewer goods than factors $(n<f){ }^{2}$ The first case is typical in empirical applications. in which case it takes the form:

$$
A=\left[u_{1}|\cdots| u_{n}\right]\left[\begin{array}{ccc}
s_{1} & \ldots & 0 \\
0 & \ddots & 0 \\
0 & \cdots & s_{f} \\
0 & \cdots & 0 \\
0 & \cdots & 0
\end{array}\right]\left[\begin{array}{c}
v_{1}^{T} \\
\vdots \\
v_{f}^{T}
\end{array}\right]
$$

\footnotetext{
${ }^{1}$ This decomposition is not unique; in particular the formula works just as well with $-U$ and $-V$. The choice of eigenvectors when there are repeat eigenvalues and the ordering of the columns of these matrices may also be arbitrary.

${ }^{2}$ Notice that I am making no assumptions about the rank of $A(w)$. Some of the singular values may be zero.
} 
In the even case, studied so extensively in the literature and taught in our textbooks, $U, S$, and $V$ are all square matrices of size $n \times n$. If there are more factors than goods, one may write:

$$
A=\left[u_{1}|\cdots| u_{n}\right]\left[\begin{array}{cccccc}
s_{1} & \cdots & 0 & 0 & \cdots & 0 \\
0 & \ddots & 0 & 0 & \cdots & 0 \\
0 & \cdots & s_{n} & 0 & \cdots & 0
\end{array}\right]\left[\begin{array}{c}
v_{1}^{T} \\
\vdots \\
v_{f}^{T}
\end{array}\right]
$$

\section{Factor prices and output prices}

Let $p$ be the $n \times 1$ vector of goods prices. Assuming that each good is produced at local unit cost, ${ }^{3}$ one may write:

$$
p=A w=U S V^{T} w
$$

This expression shows that goods prices can be computed from factor prices in three steps. First, $V^{T}$ computes a change of coordinates for factor prices. ${ }^{4}$ Second, $S$ expands or shrinks these "rotated" factor prices. Third, $U$ takes these rotated and dilated factor prices and rotates them into goods prices. In the usual case where $n>f$, this last step embeds factor prices into goods prices.

Following this intuition and recalling that $U^{T}=U^{-1}$, I let the expressions $U^{T} p$ and $V^{T} w$ define the prices of $n$ composite goods and $f$ composite factors. Write these prices as $\tilde{p}=U^{T} p$ and $\tilde{w}=V^{T} w$ respectively. They satisfy:

$$
\tilde{p}=S \tilde{w}
$$

In the case where $n>f$, equation (2) shows that the last $n-f$ of these composite goods prices are zero, expressing the restriction on prices $p$ that keep all $n$ industries competitive. Consider arbitrary changes in goods prices $d p \in \mathbb{R}^{n}$. Then $d p_{i}=0$ for $i=f+1, \ldots, n$ is an explicit restriction on the Stolper-Samuelson effects when $n>f$. These restrictions are the $n-f$ eigenvectors in the columns of $U$ that correspond to the zero eigenvalues arising because $A A^{T}$ is singular.

Consider such an eigenvector $u_{i}$. Hence, $A A^{T} u_{i}=0$. The quadratic form

$$
u_{i}^{T} A A^{T} u_{i}=\left(A^{T} u_{i}\right)^{T} A^{T} u_{i}=0
$$

\footnotetext{
${ }^{3}$ If local unit cost exceeds price, then there is no output in that sector, and one may as well delete that row of the technology matrix.

${ }^{4}$ Since $V$ is a unitary matrix, $V^{T}=V^{-1}$. Some readers might prefer to think of this change of coordinates as unraveling the given factor prices into ones that have deeper economic meaning.
} 
implies that $A^{T} u_{i}=0$. Hence, $u_{i}$ has no factor content for the given technology matrix. Every such vector lies in the left null space of $A(w)$. These vectors describe the flats along the production possibility frontier.

The condition that $A^{T} u_{i}=0$ actually has a deeper economic intuition: the eigenvector $u_{i}$ is orthogonal to goods prices. Let $p=A w$. Then

$$
p^{T} u_{i}=w^{T} A^{T} u_{i}=0 .
$$

Every such eigenvector is orthogonal to the given output price vector. Hence, the flats on the production possibility frontier are described by the last $n-f$ columns of $U$, and they form an orthonormal basis for the space of output changes that hold the value of national output constant. The columns of $U$ are an orthonormal basis for all of $\mathbb{R}^{n}$, and, when the technology matrix has full rank, they give an orthogonal decomposition of that space into $f$ vectors that span the space of output prices, and $n-f$ vectors that span the left null space of the technology matrix. ${ }^{5}$

Here is another way of understanding the implication of equation (2). The composite commodities' prices satisfy:

$$
\tilde{p}=U^{T} p=U^{T} A w=\left[\begin{array}{c}
u_{1}^{T} \\
\vdots \\
u_{f}^{T} \\
u_{f+1}^{T} \\
\vdots \\
u_{n}^{T}
\end{array}\right] A w=\left[\begin{array}{c}
u_{1}^{T} A \\
\vdots \\
u_{f}^{T} A \\
0^{T} \\
\vdots \\
0^{T}
\end{array}\right] w .
$$

This equation shows elegantly that the composite commodity prices retains exactly the right amount of information inherent in factor prices. Only the first $f$ composite commodity prices are not zero because only $f$ factors prices determine all $n$ goods prices completely.

Again, the case where $n=f$ has traditionally been called "even". $S$ is now square, and there is a very simple relationship between the composite factor prices and the composite goods prices. Each composite factor is identified with its own composite good. All of the usual Stolper-Samuelson effects are captured completely by the definitions of the composite factors and commodities!

The case where $n<f$ characterized by (3) occurs, for example, when there are some specific factors. There are more factor prices than there are goods prices,

\footnotetext{
${ }^{5}$ If $A(w)$ does not have full rank, this decomposition is still valid, but fewer than $f$ of the singular values are not zero, and the left null space of $A(w)$ has dimension greater than $n-f$.
} 
and there are more composite factor prices than there are composite goods prices. Now factor prices are not injective; different factor prices can give rise to the same unit costs. In this case, there is no implicit restriction on output prices because many different factor prices give rise to the same goods prices.

In the case represented by (3), goods prices alone do not determine factor prices; they are pinned down by other considerations, such as local endowments. Indeed, if production functions are differentiable, then factor prices are not even locally independent of endowments. Still, the special case of a Leontief technology allows for different factor prices supporting the same unit costs, even when these factor prices are independent of endowments. In this case, there are too many composite factor prices. The last $f-n$ such prices are restrictions on factor prices that allow the economy to maintain full employment.

Of course, now $A^{T} A$ is singular. The next section shows that this fact puts an explicit restriction on endowments that keeps all factor supplies in fixed proportions. This restriction makes Rybczynski effects well defined because only then are marginal changes in endowments consistent with fixed factor prices.

\section{Factor content and output vectors}

We have studied the relationship between factor prices and goods prices. This section-by contrast-concentrates on the relationship between quantities. I am now concerned with output vectors and endowments of factors. The essence of the analysis is that equations (2) and (3) are now reversed. When there are more goods than factors, only factor content is well defined. Also, when there are more factors than goods, Rybczynski effects make sense only if marginal changes in factor endowments satisfy extra restrictions inherent in the technology matrix.

The $f \times 1$ vector of factor content $x$ of the $n \times 1$ vector of output $y$ is: $^{6}$

$$
x=A^{T} y=V S^{T} U^{T} y
$$

Defining a vector of composite commodities $\tilde{y}=U^{T} y=U^{-1} y$ and one of composite factors $\tilde{x}=V^{T} x=V^{-1} x$, one may write:

$$
\tilde{x}=S^{T} \tilde{y}
$$

All the properties of the composite commodities and composite factors explored in the last section are still here in these definitions.

\footnotetext{
${ }^{6} \mathrm{I}$ am assuming that the shadow value of every factor is strictly positive and the full employment condition holds with equality.
} 
If $n>f$, then $S^{T}$ has a right block of zeros. This is the typical case used in the empirical work. Now only the first $f$ composite commodities matter in computing the factor content of an arbitrary vector of output. The intuition is that the last $n-f$ composite commodities have no factor content. Again, let $u_{i}$ be one to the last $n-f$ columns of $U$, and recall that

$$
A^{T} u_{i}=0
$$

The last $n-f$ columns of $U$ are an orthonormal basis for the null space of $A^{T}$. They are the formalization of the idea of Vanek (1968) that trade in goods is really a veil for the trade in the underlying factors used to make them.

If $n=f$, then again there is a very simple relationship between the composite factors and the composite goods.

If $n<f$, then $S^{T}$ has a lower block of zeros, and the last $f-n$ composite factors are identically zero. These equations define linear restrictions in the space of factors under which factor prices are constant and Rybczynski effects are well defined. Since factor prices are not independent of factor endowments when production functions are differentiable, these equations indicate marginal changes in endowments that keep factor prices fixed.

What is the intuition? In this case, $A^{T} A$ is singular, and the last $f-n$ columns of $V$ are eigenvectors with zero eigenvalues. Let $v_{i}$ be such an eigenvector. Then $A^{T} A v_{i}=0$. Since the quadratic form

$$
v_{i}^{T} A^{T} A v_{i}=\left(A v_{i}\right)^{T} A v_{i}=0,
$$

one may conclude that $A v_{i}=0$. Hence $v_{i}$ has no output cost in any sector, given the technology $A(w)$. The set of these vectors form an orthonormal basis for the space of factor price changes that keep all unit costs constant.

Now let $d y \in R_{+}^{n}$ be any marginal change in output. Multiplying on the left by $d y^{T}$ and using the full employment condition for fixed factor prices, we derive:

$$
d y^{T} A v_{i}=d x^{T} v_{i}=0 .
$$

Each $v_{i}$ is orthogonal to the change in economy's endowments. These shadow values show marginal changes in endowments that hold national income constant.

Here is another way of grasping the economic intuition inherent in the fact that $A v_{i}=0$ for the last $f-n$ columns of $V$. Using the full employment condition, 
we see that the definition of the composite factors satisfies:

$$
\tilde{x}=V^{T} x=V^{T} A^{T} y=\left[\begin{array}{c}
v_{1}^{T} \\
\vdots \\
v_{n}^{T} \\
v_{n+1}^{T} \\
\vdots \\
v_{f}^{T}
\end{array}\right] A^{T} y=\left[\begin{array}{c}
v_{1}^{T} A^{T} \\
\vdots \\
v_{n}^{T} A^{T} \\
0^{T} \\
\vdots \\
0^{T}
\end{array}\right] y
$$

The last $f-n$ equations are restrictions on the full employment of all factors. No matter what output vector $y$ is given, the last $f-n$ composite factors supplies will always be zero. Only endowment vectors that are orthogonal to the last $f-n$ eigenvectors in $V$ are consistent with the given factor prices $w$ and its technology matrix $A(w)$. Also, the only Rybczynski effects that make sense are endowment changes that are orthogonal to $v_{i}$ for $i=n+1, \ldots, f$. In particular, the textbook treatment of the comparative statics of the specific factors model necessarily confounds Rybczynski effects with factor price changes that maintain full employment. If he supply of a single specific factor or mobile to changes, the economy's factor prices must automatically adjust.

\section{Interpreting the singular value decomposition}

Let $A(w)$ have rank $r \leq \min \{n, f\}$. The singular value decomposition shows $A$ is the sum of $r$ different matrices, each of rank one:

$$
A(w)=\sum_{i=1}^{r} u_{i} s_{i} v_{i}^{T}=\sum_{i=1}^{r} A_{i}
$$

where again $u_{i}$ is the $i-t h$ column of $U, s_{i}$ is the corresponding singular value, and $v_{i}$ is the analogous column of $V$. This decomposition suppresses the fact that these scalars, vectors, and matrices all depend upon factor price. Thus any technology matrix can be decomposed into the sum of at most $r$ matrices of the form $A_{i} \equiv u_{i} s_{i} v_{i}^{T}$. The index of separability

$$
\mu_{i}=\frac{s_{i}^{2}}{\sum_{i=i}^{f} s_{i}^{2}},
$$

shows the fraction of the power in $A$ explained by $A_{i}$. 
The term $s_{i}$ is the inverse of a Hicks-neutral technological parameter. A larger value of $s_{i}$ raises the unit input requirements of every factor in every sector. The elements of $u_{i}$ are the inverses of sector-specific total factor productivities. In a technology matrix of rank one, $u_{i}$ is collinear with the $n$ goods prices. If one of the elements of this vector is large, then that sector requires more of every input. The elements of $v_{i}$ are the inverses of factor-specific productivities. If one of its elements is large, then that factor is not very productive.

An important insight is that relative output prices are independent of factor prices, if the technology matrix has rank one. Recall the fundamental relation: $p=A w$, where again $p$ is the $n \times 1$ vector of goods prices and $w$ is the $f \times 1$ vector of factor prices. If $A$ is of rank 1 , then it is of the form $A=u s v^{T}$. Then $p=u s v^{T} w$ and $p_{i} / p_{j}=u_{i} / u_{j}$, which does not depend upon $w$.

The full employment condition is: $x=A^{T} y$, where again $x$ is the $f \times 1$ vector of endowments and $y$ is the $n \times 1$ vector of outputs. If $A$ has rank one, the economy's aggregate factor intensities are independent of the sectoral composition of output. If $A$ has rank one, $S$ is a scalar $s$ and full employment satisfies $x=s v u^{T} y$. We infer that $x_{i} / x_{j}=v_{i} / v_{j}$ and the aggregate ratio of factor $i$ to factor $j$ does not depend upon the mix of goods being produced. This is a generalization of how trade economists think of a Ricardian technology.

An interesting area of empirical research would be to compute indices of separability for technology matrices for different countries. These matrices are now available for a wide sample of countries, and they are measured consistently and without error. One could ask a simple question, "How much of the power in the technology matrix for the United States is explained by the first component of its singular value decomposition?" If the index of separability for $\mu_{1}$ were near $100 \%$, then variability in factor uses by sector would not be empirically significant.

Using the data from Fisher \& Marshall (2013), I computed the singular value decomposition for the United States technology matrix in $2005 .{ }^{7}$ That $37 \times 5$ matrix gives the direct and indirect cost shares by industry for these factors: land, natural resources, capital, labor, and social capital. The first component explains 93\% of the variability in the data! It has these factor costs shares:

$$
v_{1}^{T}=(0.02,0.03,0.33,0.56,0.06) .
$$

The first component computes economy-wide cost shares of $2 \%$ to land, $3 \%$ to natural resources, $33 \%$ to capital, and $56 \%$ to labor. An examination of $u_{1}$ show that the most productive industry is mining, the second is agriculture, and the third

\footnotetext{
${ }^{7}$ I would like to thank the referee who suggested that I included this brief empirical exercise.
} 
is refined petroleum products. I hope this cursory description whets the reader's appetite for future empirical research.

\section{Rybczynski effects}

Fisher \& Marshall (2011) show that the Moore-Penrose inverse of the transposed technology matrix is the Rybczynski matrix for this economy. It maintains the relationship between the Stolper-Samuelson effects and the Rybczynski effects that is at the heart of trade theory. The singular value decomposition is perhaps most useful in computing the generalized inverse of a matrix. In particular,

$$
A^{+}=V S^{+} U^{T}
$$

where $S^{+}$is the generalized inverse of $S .{ }^{8}$ This inverse has a very simple form. Indeed, when $n>f$ and $s_{i}>0$ for all $i$,

$$
S^{+}=\left[\begin{array}{cccccc}
1 / s_{1} & \ldots & 0 & 0 & \ldots & 0 \\
0 & \ddots & 0 & 0 & \ldots & 0 \\
0 & \ldots & 1 / s_{f} & 0 & \ldots & 0
\end{array}\right]
$$

The other case is completely analogous.

This structure shows that the canonical sign pattern actually depends upon the lower order matrices in the decomposition. The first term $v_{1}\left(1 / s_{i}\right) u_{1}^{T}$ will almost always have all positive elements. Hence it is impossible for a good to be the enemy of any factor if a technology matrix has rank one. Only when we get to the small singular values will some element of $v_{i}\left(1 / s_{i}\right) u_{i}^{T}$ be negative. Notice that it is also necessary for the singular value $s_{i}$ to be small enough so that $1 / s_{i}$ is large. That is why almost singular technology matrices have strong Rybczynski effects. But a component of the singular value decomposition where $s_{i}=0$ will have no effect on the Rybczynski matrix because $s_{i}^{+}=0$ for that part of the effect.

This observation has important implications for the empirical literature on estimating Rybczynski effects. The original idea is due to Rybczynski (1955), and recent empirical applications can be found in Harrigan (1995), Schaur et al. (2008), and Romalis (2004). The key insight is that the negative elements in any singular value decomposition will be associated typically with the smaller singular values, since the technology matrix itself consists of all non-negative elements.

\footnotetext{
${ }^{8}$ Let $s_{i}^{+}=s_{i}^{-1}$ if $s_{i} \neq 0$ and $s_{i}^{+}=0$ if $s_{i}=0$. Then $S^{+}$is the natural diagonal matrix that has the same dimensions as $S^{T}$.
} 
The typical model studied in empirical trade has $n>f$. In this case, most trade theorists argue that Rybczynski effects are not well defined. Still, the typical empirically oriented trade economist runs regression of output vectors on endowments and claims to have identified Rybczynski effects; of course these regressions are not even identified under the null. Indeed, it is easy to show that the typical Rybczynski regression actually estimates demand parameters that have nothing to do with general equilibrium supply effects. Fisher \& Marshall (2011) show how to calculate-not estimate-the Rybczynski matrix given data on the local technology matrix. But the negative elements that identify a factor's enemies come from the smallest singular values. So if a technology matrix is measured with error, its Rybczynski matrix may be imprecisely calculated.

\section{Examples from the three canons of trade theory}

I shall now go through the three simplest cases that capture all of trade theory. The first is the simplest case with more goods factors than goods: the Solow growth model with aggregate output and capital and labor as inputs. The second is the textbook two-sector model, with two goods and two factors; it shows the power of my decomposition forcefully. The third case is the Ricardian model where there are $n$ goods and one composite factor. I shall derive algebraic expressions for the singular value composition in every case.

\subsection{More factors than goods}

The canonical case is the Solow model used in macro with technology matrix:

$$
A=\left[\begin{array}{ll}
a_{K} & a_{L}
\end{array}\right]
$$

where the first column corresponds to inputs of capital and the second to that of labor. The eigenvalue of $A A^{T}$ is obviously $a_{K}^{2}+a_{L}^{2}$, so the first singular value is $\sqrt{a_{K}^{2}+a_{L}^{2}}$. In fact,

$$
\begin{gathered}
U=[1], \\
S=\left[\begin{array}{ll}
\sqrt{a_{K}^{2}+a_{L}^{2}} & 0
\end{array}\right],
\end{gathered}
$$

and

$$
V=\left[\begin{array}{cr}
a_{K} / \sqrt{a_{K}^{2}+a_{L}^{2}} & a_{L} / \sqrt{a_{K}^{2}+a_{L}^{2}} \\
a_{L} / \sqrt{a_{K}^{2}+a_{L}^{2}} & -a_{K} / \sqrt{a_{K}^{2}+a_{L}^{2}}
\end{array}\right] .
$$


In this case, the composite commodity is trivial since there is only one good. Hence $\tilde{p}=p$. The first composite factor-defined by the first column of $V-$ combines capital and labor, according to the economy's aggregate capital-labor ratio. The second column of $V$ states that the economy's capital-labor ratio must insure full employment of all factors at the given factor prices. This is the meaning of the constraint inherent in the definition of the second composite factor: $\tilde{x}_{2}=v_{2}^{T} x=v_{2}^{T} A^{T} y=0$ for any quantity of output $y>0$. The matrix $V$ is uniquely defined in this case by the fact that there is only a one-dimensional null space for $A$; if there were three or more factors, then there would be some ambiguity in defining the orthonormal basis for the (right) null space of $A$.

\subsection{The Even Case}

Now consider the textbook case:

$$
A=\left[\begin{array}{ll}
a_{1 K} & a_{1 L} \\
a_{2 K} & a_{2 L}
\end{array}\right] .
$$

I shall explore three canonical cases. The simplest is:

$$
A=\left[\begin{array}{cc}
a_{1 K} & 0 \\
0 & a_{2 L}
\end{array}\right] .
$$

This matrix has full rank, but each sector uses only one factor. Then

$$
\begin{gathered}
U=\left[\begin{array}{ll}
1 & 0 \\
0 & 1
\end{array}\right], \\
S=\left[\begin{array}{rr}
a_{1 K} & 0 \\
0 & a_{2 L}
\end{array}\right],
\end{gathered}
$$

and

$$
V=\left[\begin{array}{ll}
1 & 0 \\
0 & 1
\end{array}\right] \text {. }
$$

The composite factors and composite commodities are defined exactly as one might expect. This is the only case where the composite commodities and the composite factors are just the actual commodities and the actual factors themselves. The Stolper-Samuelson effects are:

$$
A^{+}=V S^{+} U^{T}=S^{-1}=\left[\begin{array}{rr}
1 / a_{1 K} & 0 \\
0 & 1 / a_{2 L}
\end{array}\right],
$$


and a strong effect occurs in a sector that uses little of the requisite input.

The singular value decomposition even works in the case where the technology matrix is singular! Consider

$$
A=\left[\begin{array}{cc}
\theta_{1} a_{K} & \theta_{1} a_{L} \\
\theta_{2} a_{K} & \theta_{2} a_{L}
\end{array}\right]
$$

for (inverse) total factor productivity parameters $\theta_{1}>0$ and $\theta_{2}>0$; the two sectors have identical capital-labor ratios. Since $A$ is singular, so is $A^{T} A$. Indeed, the determinant of $A^{T} A$ is 0 , and its trace is $\Theta \equiv\left(\theta_{1}^{2}+\theta_{2}^{2}\right)\left(a_{K}^{2}+a_{L}^{2}\right)$. So its first singular value is $\sqrt{\Theta}$ and its second is 0 . One may compute:

$$
\begin{gathered}
U=\left[\begin{array}{cc}
\theta_{1} / \sqrt{\theta_{1}^{2}+\theta_{2}^{2}} & -\theta_{2} / \sqrt{\theta_{1}^{2}+\theta_{2}^{2}} \\
\theta_{2} / \sqrt{\theta_{1}^{2}+\theta_{2}^{2}} & \theta_{1} / \sqrt{\theta_{1}^{2}+\theta_{2}^{2}}
\end{array}\right], \\
S=\left[\begin{array}{rr}
\sqrt{\Theta} & 0 \\
0 & 0
\end{array}\right],
\end{gathered}
$$

and

$$
V=\left[\begin{array}{cc}
a_{K} / \sqrt{a_{K}^{2}+a_{L}^{2}} & -a_{L} / \sqrt{a_{K}^{2}+a_{L}^{2}} \\
a_{L} / \sqrt{a_{K}^{2}+a_{L}^{2}} & a_{K} / \sqrt{a_{K}^{2}+a_{L}^{2}}
\end{array}\right] .
$$

The first column of $U$ shows composite commodity consistsing of fixed amounts of the first good and second good, where the weighting scheme depends upon a natural combination of the total factor productivities. In particular, the sector that uses a lot of resources gets a big weight. The second column of $U$ shows that the relative price of the two goods must reflect total factor productivities. The price of the second composite commodity defines the constraint: $\tilde{p}_{2}=-\theta_{2} p_{1}+\theta_{1} p_{2}=0$. The first column of $V$ shows that the composite factor consists of exactly the capital and labor that is used in every sector. The second column of $V$ shows that the economy can maintain full employment only if its endowment satisfies the requisite capital-labor ratio, given the factor prices that define the technology matrix implicitly. Notice that this is the same full employment constraint for the case where there is one good and two factors.

There may not be much to be gained by analyzing the general case where the technology matrix $A$ has full rank and is not diagonal, but it is perhaps worthwhile to close this subsection by examining a nearly singular a technology matrix:

$$
A=\left[\begin{array}{cc}
1 & 1 \\
1.01 & 1
\end{array}\right]
$$


Computation shows that:

$$
\begin{gathered}
U=\left[\begin{array}{rr}
-0.7053 & -0.7089 \\
-0.7089 & 0.7053
\end{array}\right], \\
S=\left[\begin{array}{rr}
2.005 & 0 \\
0 & 0.005
\end{array}\right],
\end{gathered}
$$

and

$$
V=\left[\begin{array}{rr}
-0.7089 & 0.7053 \\
-0.7053 & -0.7089
\end{array}\right]
$$

One can now see the utility of the decomposition:

$$
A=u_{1} s_{1} v_{1}^{T}+u_{2} s_{2} v_{2}^{T}=\left[\begin{array}{ll}
1.0025 & 0.9975 \\
1.0075 & 1.0025
\end{array}\right]+\left[\begin{array}{cc}
-0.0025 & 0.0025 \\
0.0025 & -0.0025
\end{array}\right] .
$$

The first singular value explains $\mu_{1}=s_{1}^{2} /\left(s_{1}^{2}+s_{2}^{2}\right) \approx 100 \%$ of the variability in the data. For all practical purposes, the technology $A$ is completely explained by $A_{1}=u_{1} s_{1} v_{1}^{T}$. This singular matrix has an identical capital-labor ratio in both sectors of $1.0051=0.7089 / 0.7053$, and the two sectors have slightly different (inverse) total factor productivities whose ratio is given by $0.9949=0.7053 / 0.7089$. The practical importance of this distinction is highlighted when one contrasts the Stolper-Samuelson matrix:

$$
A^{-1}=\left[\begin{array}{cc}
-100 & 100 \\
101 & -100
\end{array}\right]
$$

with

$$
A_{1}^{+}=\left[\begin{array}{ll}
0.2494 & 0.2506 \\
0.2481 & 0.2494
\end{array}\right]
$$

where $A_{1} \equiv u_{1} s_{1} v_{1}^{T}$. If an empirical trade economist is worried that she has measured $A$ with slight error, she would compute very large Stolper-Samuelson effects according to $A^{-1}$. If she came to the guarded empirical conclusion that both sectors had identical capital-labor ratios, then the proper interpretation of these effects is given by $A_{1}^{+}$, and it is very different indeed. In particular, it might not be warranted to state that the first sector is a strong enemy of capital and the second a strong enemy of labor. 


\subsection{More goods than factors}

The Ricardian model is the simplest case where $n>f$. The technology matrix

$$
A=\left[\begin{array}{c}
a_{1} \\
\vdots \\
a_{n}
\end{array}\right]
$$

has full rank as long as some $a_{i} \neq 0$. Let $a=a_{1}^{2}+\cdots+a_{n}^{2}$. Then the singular value decomposition of this technology matrix satisfies:

$$
\begin{gathered}
U=\left[\begin{array}{cccc}
a_{1} / \sqrt{a} & u_{12} & \cdots & u_{1 n} \\
\vdots & \vdots & \vdots & \vdots \\
a_{n} / \sqrt{a} & u_{n 2} & \cdots & u_{n n}
\end{array}\right] \\
S=\left[\begin{array}{c}
\sqrt{a} \\
0 \\
\vdots \\
0
\end{array}\right]
\end{gathered}
$$

and

$$
V=[1] \text {. }
$$

Now there is a single composite factor called "labor". The rank of $A A^{T}$ is $f=1$ in this case, and the $n-1$ orthonormal eigenvectors in $U$ are not unique. Hence the composite commodity

$$
\tilde{y}=U^{T} y
$$

is not unique either. Still, the last $n-1$ elements of $\tilde{y}$ have no factor content.

Let $w>0$ be a given shadow value for the primary factors. The vector of composite commodity prices is:

$$
\tilde{p}=U^{T} p=U^{T} A w=\left[\begin{array}{c}
A^{T} / \sqrt{a} \\
u_{2}^{T} \\
\vdots \\
u_{n}^{T}
\end{array}\right] A w=\left[\begin{array}{c}
w \sqrt{a} \\
0 \\
\vdots \\
0
\end{array}\right] .
$$

The last $n-1$ columns of $U$ give a basis for the space of all vectors in $\mathbb{R}^{n}$ that are orthogonal to the linear production possibility frontier that is the essence of 
a Ricardian technology. These composite commodity prices are perhaps best interpreted as restrictions on local price changes $d p$ that allow all sectors to remain competitive on world markets. The basis for these changes is not unique, but the decomposition of $\mathbb{R}^{n}$ into the subspace spanned by vector $\left(a_{1} / \sqrt{a}, \cdots, a_{n} / \sqrt{a}\right)^{T}$ and its orthogonal complement is.

\section{Conclusion}

Jones (1965) was far sighted to emphasize that the essence of international trade theory is the study of simple models of general equilibrium. For local changes in factor prices, the mathematics of these models is the study of matrices with nonnegative elements; the deep structure of international trade theory is applied linear algebra. The singular value decomposition is perhaps one of the most elegant tools in modern linear algebra, and it used widely in the natural sciences. For example, it has been applied in genetics by Alter et al. (2000), oceanography by Wallace et al. (1992), and image processing by Boardman (1989). It has begun to be used in econometrics by Kleibergen \& Paap (2006). I have given its first use in international economics.

Any technology matrix serves two purposes: (1) it is a linear mapping of local factor price changes into local output price changes; and (2) for fixed output prices and thus factor prices, it is a linear mapping between changes in endowments and changes in outputs. These two approaches are mathematically dual; this was the deep insight of Samuelson (1953), based upon his earlier co-authored work Stolper \& Samuelson (1941). This duality is most easily seen in an even model, where the number of factors is equal to that of goods. But such models are far from general, and they have limited empirical application. Trade theorists have generalized the canonical $2 \times 2$ model at least the classic work of Ethier (1974).

I have played two leitmotifs. The first is that the singular value decomposition carries useful information when the number of goods is not equal to that of factors. When there are more factors than goods, the restrictions on factor prices that maintain full employment can be read from the last $f-n$ columns of $V$. When there are more goods than factors, as is typical in almost every empirical application, then the last $n-f$ columns of $U$ give restrictions on changes in output prices that allow all sectors to remain active. These restrictions are inherent in our definitions of the composite commodities and factors. The second is the duality between prices and quantities that is inherent in a proper interpretation of the comparative statics of simple models general equilibrium. 
Applications of these ideas in computing Rybczynski effects using only the first one or two singular values of measured technology matrices may be useful. I have shed the first light on an attractive alternative to imposing a translog functional form as an approximation of the national revenue function, the typical careful empirical application of trade theory to the data. Since technology matrices are now measured consistently for many countries, there are many ready and fruitful empirical applications of these ideas. It is important to know how nearly singular any technology matrix is, and these ideas can be readily applied in the usual case where there are many more goods than factors.

\section{References}

Alter, O., Brown, P. \& Botstein, D. (2000), 'Singular value decomposition for genome-wide expression data processing and modeling', Proceedings of the National Academy of Sciences 97(18), 10101-10106.

Boardman, J. (1989), 'Inversion of imaging spectrometry data using singular value decomposition', Quantitative remote sensing: An economic tool for the Nineties pp. 2069-2072.

Chang, W. W., Ethier, W. J. \& Kemp, M. C. (1980), 'The theorems of international trade with joint production', Journal of International Economics 10(3), 377394.

Ethier, W. (1974), 'Some of the theorems of international trade with many goods and factors', Journal of International Economics 4, 199-206.

Ethier, W. J. (1984), 'Higher dimensional issues in trade theory', Handbook of international economics 1, 131-184.

Fisher, E. O. \& Marshall, K. G. (2011), 'The Structure of the American Economy', Review of International Economics 19, 15-31.

Fisher, E. O. \& Marshall, K. G. (2013), 'Testing the Heckscher-Ohlin-Vanek Paradigm in a World With Cheap Foreign Labor', Unpublished manuscript.

Harrigan, J. (1995), 'Factor endowments and the international location of production: Econometric evidence for the oecd, 1970-1985', Journal of International Economics 39(12), 123 - 141. 
Jones, R. (1965), 'The structure of simple general equilibrium models', The Journal of Political Economy 73(6), 557-572.

Kleibergen, F. \& Paap, R. (2006), 'Generalized reduced rank tests using the singular value decomposition', Journal of Econometrics 133(1), 97-126.

Romalis, J. (2004), 'Factor Proportions and the Structure of Commodity Trade', American Economic Review 94(1), 67-97.

Rybczynski, T. M. (1955), 'Factor endowment and relative commodity prices', Economica 22(88), pp. 336-341.

Samuelson, P. A. (1953), 'Prices of factors and goods in general equilibrium', Review of Economic Studies 21(1), 1-20.

Schaur, G., Xiang, C. \& Savikhin, A. (2008), 'Factor uses and the pattern of specialization', Review of International Economics 16(2), 368-382.

Stolper, W. F. \& Samuelson, P. A. (1941), 'Protection and real wages', Review of Economic Studies 19, 58-73.

Vanek, J. (1968), 'The factor proportions theory: The n-factor case', Kyklos 21(4), 749-756.

Wallace, J., Smith, C., Bretherton, C. et al. (1992), 'Singular value decomposition of wintertime sea surface temperature and 500-mb height anomalies', Journal of climate 5(6), 561-576. 